\title{
Mesure des débits de la Basse-Seine
}

\section{Discharge measurements of the lower Seine}

\author{
M. BLOSELT

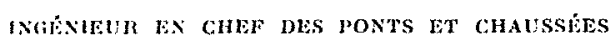 \\ CHEF DU SETVICE DE LA NAVIGATION \\ DE LA BASSF-SEINE
}

\author{
PAR \\ BT
$\dagger$ M. FOUILLET (1)
INGËNIELTR DES T.P.J. lat HYDROLOCHQUeS DU SEHVICE

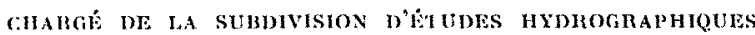

\begin{abstract}
Les profondes modifications exéculées sur la Busse-Seine depnis 1930, et non encore achevées, ont rendu caduques les formules empiri'ques employées jusqu'à présent pour calculer les débits de ce fleuve en fonction de la cote lue en rivière libre. Aussi, le Service de la Navigation a-t-il da procéder a des jaugeages au moulinet dans la région Bougival-Croissy, sur deux bras partiellement interdits à la navigation, en vue de tarer une station permanente $\dot{a}$ deux limnigraphes distants de 12 kilomètres dans un bief de la Seine éloigné des ouvrages de navigation. A l'étiage, la pente trop faible ne permettant pas d'utiliser la station, on détermine les faibles debits en étalonnant les barrages voisins.
\end{abstract}

\begin{abstract}
The extensive modifications which have been made to the lower Seine since 1930, and which are still not complete, have outdated the empirical formulae, depending on the recorded levels of the free water surface, which have been used up to now for calculating the discharge of the river. For this reason the Service de la Navigation has had to carry out current meter gangings in the Bougival-Croissy region, on two branches partly closed to navigation. These readings will serve to calibrate a permanent station comprising two limnigraphs 12 kilometers apart on a reach of the Seine some distance away from navigation worlss. When the Seine is at its lowest, its slight slope isn't sufficient for the station to be used, so the small discharges are determined by calibraling the local barrages.
\end{abstract}

\section{INTRODUCTION ET HISTORIQUE SUCCINCT}

Il ne semble pas que des études systématiques aient été faites dans le passé quant à l'appréciation des débits d'étiage de crues, dans la partie de la Seine située entre l'aval de Paris et le dernier barrage limitant la canalisation du fleuve, Martot jusqu'en 1939, Poses depuis cette date.

(1) En publiant cette note, c'est pour nous un bien triste devoir de rendre un hommage ému à la mémoire de l'un de ses auteurs, M. Fovilner, qui avait présenté au Comité Technique, le 10 juin 1954, la communication dont elle a fait l'objet.

Encore jeune et promis à un avenir plein d'espérance, M. Founleter disparaît prématurément après une courte maladie. Il est profondément regretté de ses chefs, de ses collègues et de tous les membres de la S.H.F. qui avaient l'heur de le connaitre.

N.D.L.R
Certes, quelques mesures de vitesse avaient éte effectuées au droit de tel ou tel ouvrage, pont ou barrage effacé, notamment en 1876, 1910, 1924, $1926 \ldots$ et des formules de débits plus ou mons empiriques avaient été présentées à l'époque, dont certaines figurent encore dans les traités classiques, telle celle énoncée par de Préaudear (1876) pour le débit de la Seine à Mantes :

$$
\mathrm{Q}=170+150(h-0,8)+22(h-0,8)^{2}
$$

$h$ représentant la cote lue en "rivière libre $», a$ l'échelle du vieux pont de Mantes, échelle dont le zéro, d'abord rapporté en nivellement Bourdaloue, est, en définitive, situé à l'altitude (+ 12,37) par rapport au zéro du N.G.F. (LaLli:MAND). 
L'auteur signalait d'ailleurs que sa formule n'était valable que jusqu'à la limite de $800 \mathrm{~m}^{3} / \mathrm{s}$ correspondant à l'ouverture du barrage le plus voisin, celui de Méricourt-Sandrancourt.

Plus tard, nos prédécesseurs, intéressés par le problème, reprirent la formule parabolique : $\mathrm{Q}=\mathrm{A}+\mathrm{B}(h-a)+\mathrm{C}(h-a)^{2}$, et essayèrent de fixer des coefficients convenables; nous retrouvons ainsi, dans nos archives, les propositions de M. WiLlfmin (actuellement inspecteur général des Ponts et Chaussées en retraite) pour les débits à Bezons, Mantes et Vernon, avec, pour ces deux derniers, un quatrième terme en $h^{3}$ :

$$
\begin{aligned}
& \mathrm{Q}_{\mathrm{I}}=57+100(h-0,32) \\
&+33(h-0,32)^{2} \\
& \mathrm{Q}_{\mathrm{M}}=170+140(h-0,80)+16(h-0,80)^{2}+3(h-0,80)^{3} \\
&+150(h-0,75) \\
& \mathrm{Q}_{V}=180++28(h-0,5)^{2}+4(h-0,75)^{3} \\
&
\end{aligned}
$$

Ainsi que celle de M. Lorton, ancien ingénieur des Ponts et Chaussées du Service, pour le débit de la Basse-Seine au droit du pont de SaintPierre-du-Vaurray :

$\mathrm{Q}_{s}=100+103 h+85 h^{2}$ (après développement)

En janvier 1926, d'autres mesures de vitesse, faites en crue, à Bezons et à Rangiport - amont de Mantes - conduisirent à corriger les formules précédentes et à suggérer des coefficients numériques différents :

$$
\begin{aligned}
& \text { Bezons: } \mathrm{Q}=28+56 h+28 h^{2}\left(^{*}\right) \text {. } \\
& \text { Mantes : } \mathrm{Q}=170+160(h-0,8) \\
& +16(h-0.8)^{2} \text {. } \\
& \text { 事 }
\end{aligned}
$$

Aussi longtemps que le régine du fleuve ne variait sensiblement pas, il n'y avait pas de raison de critiquer les formules ci-dessus; mais, a partir de 1930 , de profondes modifications commencèrent à ètre exćcutées dans le chenal, modifications non encore achevées actuellement, qui rendirent caducs les coefficients numériques altribués à la \& formule des Ingénieurs de la Basse-Seine $\gg$.

Citons, par ordre chronologique, la création d'un nonveau bras, dit « Rivière Neuve », entre Bezons et Bougival, avec reconstruction du barrage de Chatou (1930-1932); - le calibrage du bief de Martot comportant en particulier la sup-

(") La formule Willemin développée aurait donné :

$$
\mathrm{Q}=28+79 h+53 h^{2}
$$

pression du barrage de même nom (1932-1939); - l'aménagement du bief de Meulan avec reconstruction du barrage d'Andrésy; déplacement des écluses de Carrières-sous-Poissy à Andrésy, dragages du fleuve, etc... (1952 à ce jour); - la suppression des ouvrages de navigation de $\mathrm{Meu}-$ lan et de Port-Villez (en projet), sans parler des réalisations récentes d'E.D.F. telle la Centrale de Porcheville (amont de Mantes) dont les canaux d'amenée et de fuite modifieront notablement les conceptions antérieures en matière d'écoulement des eaux!

De nouvelles études — jaugeages principalement - devenaient indispensables :

1" Dès 1942, la Compagnie Centrale d'Energie Electrique (actuellement E.D.F. à Rouen) commençait, sous l'impulsion de son regretté président, M. Bouls, et en accord avec le Service de la Navigation de la Basse-Seine, des études dans le bief de Poses, en vue de l'installation ultérieure d'une Centrale hydroélectrique de Basse Chute; le programme, suivi d'exécution, était le suivant :

- Profil de jaugeage fixé à $300 \mathrm{~m}$ en amont du barrage (dont aucune passe n'est accessible à la navigation, en étiage comme en crue);

- Mesures au moulinet (sur bateau ou à câbles) sur sept verticales, espacées de $12,50 \mathrm{~m}$ el à des profondeurs de $0,50 \mathrm{~m}, 2,50 \mathrm{~m}, 4,50 \mathrm{~m}$, $7 \mathrm{~m}$ et $9 \mathrm{~m}$ en dessous de la retenue normale.

On ne peut que regretter que les essais, interrompus en 1944, n'aient pas élé repris depuis.

$2^{\circ}$ Au cours de la crue exceptionnelle de 1945 et à la suite de travaux importants de rescindement d'une île voisine du pont de Poissy (*), un de nos jeunes ingénieurs, actif et compétent (**), s'intéressa à la question et effectua toute une série de mesures de vitesse de courant, les unes en amont du confluent de l'Oise, au droil du pont du Pecq, les autres en aval, au droit de Poissy.

Les opérations, effectuées avec des moulinets de Woltman prêtés par la section du matériel de l'Ecole Nationale des Ponts el Chaussées, se firent à $1 \mathrm{~m}$ de profondeur, avec application systématique du coefficient de pe Précuneau de 0,8 dans la formule parabolique rappelée précédemment.

(*) D'ailleurs écrasé par les bombardements et dont le déblaiement, à l'exception des seules arches rive gauche vraiment d'époque, a été poursuivi jusqu'à ec jour.

(*) M. PLanche, maintenant ingénieur d'arrondissement a Blois. 
Les résultats, bien qu'incertains, folrnironi des renseignements intéressants, mais la nécessite de créer, dans le Service, une véritable subdivision spécialisée dans les études du régime du fleuve, dotée de personnel et de matériel moderne, devenait impérieuse.

Non sans peine, tant en raison des difficultés d'approvisionnement en matériel et matériaux que de multiples difficultés budgétaires, il nous fut possible de mettre sur pied une brigade hydrographique (ou hydrologique, selon le sens que l'on attribue aux mots), dotée d'un chef énergique et dynamique, digne successeur du précurseur cité plus haut, lequel avec une petite équipe dévouée ( 1 adjoint technique opérateur, 1 mécanicien, 1 dessinateur, quelques aides, etc.) assure depuis plusieurs années, sans arrêt, les observations hebdomadaires, sinon journalières, de la station de jaugeage créée au Pecq et commence à en exploiter les résultats.

Les pages qui suivent ont été rédigées, à notre demande, par M. Fouillet, Ingénieur T.P.E., auquel nous tenons à dire ici toute la reconnaissance du chef de service pour un travail pénible et ingrat, mais combien productif!

M. Brosset.

\section{BUTS POURSUIVIS ET PREMIERS RESULTATS ACQUIS}

Les études sur les débits des cours d'eau naturels montrent la complexité des phénomènes à considérer lorsque le cours d'eau comporte des remous provoqués par des « contrôles » naturels ou artificiels.

Sur la Seine, de nombreuses études du régime du fleuve ont été faites. Les premières tendaient uniquement à la prévision des crues. Puis la question du débit du fleuve s'est posée plus nettement et plusieurs Ingénieurs ont abouti, après étude, à proposer des formules utilisant les cotes lues à certaines échelles pour donner une estimation du débit dans la section considérée. On peut citer en particulier les travaux de MM. PoIRÉE, Cheysson, de Iagrénée et de PrÉ.ludeau.

Ces formules ont perdu toute valeur pratique actuelle par suite des importantes modifications apportées au lit du fleuve. En outre, une telle méthode d'évaluation ne peut donner une détermination correcte du débit que pour des valeurs relativement importantes. Pour les petits débits, l'imprécision de lecture du plan d'eau aux échelles ne permet même plus d'arriver à un ordre de grandeur acceptable.

De nouvelles études (MM. Gaspard el Weirich notamment) ont été effectuées depuis quelques années pour Paris et son amont. Elles ont abouti à déterminer les débits sur la base d'études théoriques et de tarages sur modèles réduits. La vérification des lois de concordance a été obtenue par un nombre limité de jaugeages réels.

Il a paru intéressant de reprendre le problème à l'envers, c'est-à-dire de créer, pour l'aval de Paris, une station de jaugeage dont le tarage serait obtenu en partant des résultats d'expérience fournis par des jaugeages réels très nombreux, de manière à établir des graphiques sûrs pour lesquels les théories de l'écoulement apporteraient seulement la possibilité de coordonner les résultats obtenus.

Cette idée a pu ètre mise en pratique dans la région Bougival-Croissy du fait de la présence en cet endroit de deux bras partiellement interdits à la navigation et permettant de jauger le

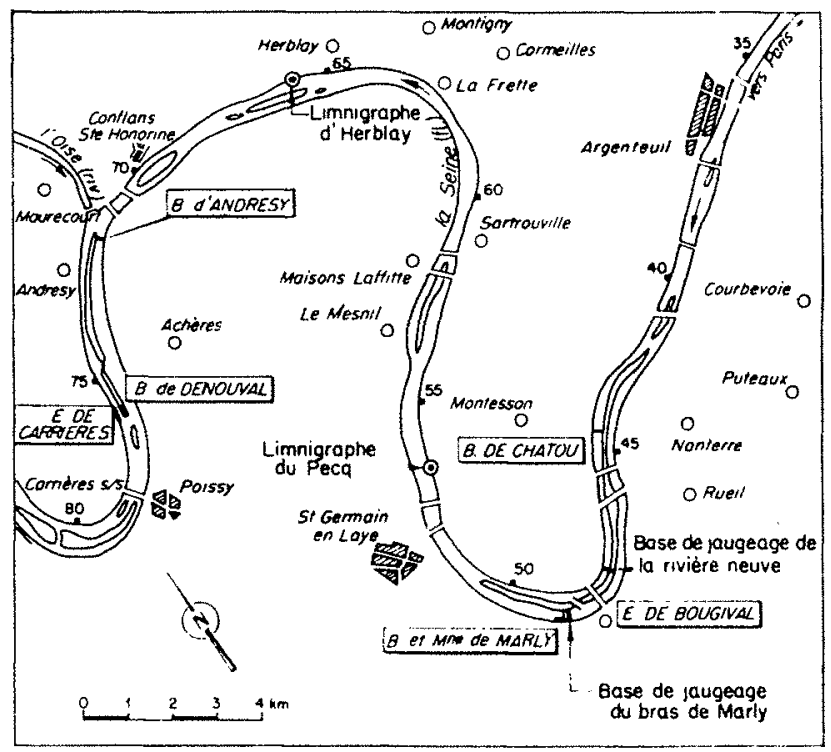

Fic. 1. .-. Emplacement des stations de jaugeage.

débit total, au moulinet, en dehors du chenal navigable (fig. 1).

Les mesures sont effectuées :

1 "Còté Croissy, dans le bras dit de la RivièreNeuve, à $1,500 \mathrm{~km}$ des écluses, dans une section interdite a la navigation en temps normal; 
$2^{\circ}$ Côté Bougival, dans le bras de Marly, au droit des écluses dans une zone interdite à la navigation en permanence.

On peut observer que le débit passant par les écluses n'est pas mesuré; il est toutefois très faible et presque constant, on peut l'évaluer à $2 \mathrm{~m}^{3} / \mathrm{s}$ environ en moyenne.

Les bases ont été implantées dans deux sections choisies en tenant compte des conditions suivantes :

Le courant doit y être exempt de remous susceptibles de fausser les mesures, condition qui impose une allure constante de la section transversale de part et d'autre de la base. La base de la «Rivière Neuve » doit en outre être suffisamment éloignée du barrage de Chatou pour ne pas subir les effets d'instabilité habituels à l'aval des chutes. Enfin, l'accès de la station doit être facile.

Les bases choisies répondent à ces différents impératifs. En particulier, la base de la « RivièreNeuve $\gg$ est implantée à près de $3 \mathrm{~km}$ du barrage de Chatou et deux ponts contribuent de plus à régulariser le courant dans la section transversale.

Les mesures sont effectuées au moulinet; l'opération dure deux heures environ. La durée assez longue de cette mesure n'offre pas d'inconvénient sur un fleuve comme la Seine où les variations de débit sont assez lentes. De plus, les mesures ne sont faites que lorsque les barrages n'ont pas modifié leur bouchure depuis au moins douze heures.

Dans les conditions actuelles, les jaugeages sont effectués toute l'année une fois par semaine. Pendant les crues, ces jaugeages sont rapprochés jusqu'à devenir journaliers pendant les périodes de débits exceptionnels.

Les résultats en sont donnés sur les courbes établies pour les années 1952 et 1953 qui seront annexées à une prochaine note.

$$
\text { *** }
$$

Ainsi qu'il vient d'être dit, ces premiers résultats doivent en outre permettre le tarage de stations permanentes susceptibles de donner les débits journaliers avec une bonne précision.

Dans ce but, une section de Seine choisie éloignée des ouvrages de navigation amont et aval, mais faisant suite aux bases de jaugeages actuelles, a été dotée de deux limnigraphes enregistreurs à une douzaine de kilomètres de distance. Les jaugeages effectués actuellement amorcent le tarage de cette section de Seine.

L'idéal serait d'obtenir par expérience un graphique couvrant tous les débits avec les cotes de contrôles possibles correspondantes.
Si l'on considère, parmi les jaugeages déjà effectués, que seuls sont à retenir, pour l'étalonnage de la station, ceux qui correspondent à une inscription sensiblement rectiligne des limnigraphes; si l'on tient compte en outre des phénomènes spéciaux aux crues et décrues qui entrainent des variations très importantes de débit pour les cotes sensiblement égales à la base, on voit que l'étalonnage de la station demande de nombreuses observations s'étendant à des cas assez rares sur la Seine.

On peut concevoir toutefois l'établissement progressif de l'abaque en sorte que prochainement les jaugeages réels des périodes les plus courantes deviendront inutiles.

Une autre difficulté, inverse de la première, se produit pour les courants d'étiage. La pente superficielle devient très faible et il est illusoire de chercher à étalonner la station pour des débits inférieurs à $150 \mathrm{~m}^{3} / \mathrm{s}$, débits pourtant assez fréquents sur la Seine.

Il est possible alors de déterminer ces faibles débits en étalonnant les barrages voisins.

Pourtant si l'opération est relativement facile au barrage de Chatou, du type à vannes Stoney avec déversement supérieur, le problème est beaucoup plus complexe au barrage de Marly dont les bouchures multiples comportent: un barrage à aiguilles, un barrage à vannettes, une machine hydraulique composée de roues à aube et de turbines. En outre, dans les périodes d'étiage qui sont surtout intéressantes pour cet étalonnage, une grande partie du débit franchit l'ouvrage par les fuiles multiples qui existent dans les installations.

Il apparaît donc comme impossible d'arriver ¿̇ un résultat correct basé sur lu seule position des organes de bouchure. On peul néanmoins arriver à un résultat acceptable au prix de quelques jaugeages test espacés de quinze jours environ, destinés à tarer les fuites de l'ouvrage. Les manœuvres sont en effet assez rares en étiage et le débit passant par les roues à aube et par les turbines peut être évalué avec une certaine précision.

Le débit passant par les ouvrages pourra done être obtenu d'une part par le calcul du débil entrainé par la position des différents organes et d'autre part par une évaluation des fuites résultant du jaugeage test.

Au demeurant, le débit d'étiage du bras de Marly varie très peu; c'est ainsi que pendant les neuf mois secs de l'année 1953, ce débit s'est tenu entre 40 et $60 \mathrm{~m}^{3} / \mathrm{s}$.

\section{**}

L'établissement progressif du tarage de la station présentera par suite les étapes successives suivantes : 
- En premier lieu, établissement des graphiques de tarages pour les débits fréquents. Ces graphiques utiliseront : les harrages pour les très faibles débits, la station à échelles pour les débits plus élevés. Une plage de recouvrement devra assurer une bonne continuité des évaluations;

- Cette première étape réalisée, les mesures pourront être centrées sur les régimes plus rares du fleuve de manière à étendre les courbes.

Une étude très intéressante pourra être effec- tuée en ce qui concerne les états non permanents de l'écoulement. Les limnigraphes enregistreurs permettront la détermination précise de la variation des niveaux par unité de temps, il sera possible de rechercher les éléments correcteurs a introduire aux graphiques pour tenir compte de la non-permanence.

En définitive, la nouvelle station de jaugeages de la région de Bougival-Croissy, tout en donnant dès maintenant les débits de la Seine en cette région, prépare l'étalonnage par l'expérience d'un système complexe dont l'étude est commencée.

\section{Foville:t.}

\section{DIS CUSSION}

M. le Président BanniLlon demande si on a enregistré les directions des courants dans la Seine pour les différents débits, par exemple en photographiant pendant la nuit les trajectoires de boućes lumineuses flottant à la surface.

M. Founllet indique que le Service de la Navigation est précisément en train de faire les enregistrements de ce genre en aval du barrage de Notre-Dame de la Garenne où il $y$ a un problème d'envasement assez important. D'autre part, il n'a pas été observé de courant traversier dans les sections des stations de jaugeage qui ont été installées sur des tronçons très rectilignes.

M. le Président remarque qu'il serait intéressant de vérifier le parallélisme des filets dans les stations de jaugeage, même lorsqu'elles sont installées dans des tronçons rectilignes.

Répondant à une question de M. Hupver, M. Fovillex précise que les points de mesure des vitesses au moulinet sont au nombre de 50 à 60 pour l'ensemble de la section de jaugeage. Ces points sont répartis, dans cette section, sur des verticales distantes de $15 \mathrm{~m}$, soit 5 stations sur le bras de Marly et 7 sur la Rivière Neuve. Chaque verticale comprend deux points de mesure, respectivement à $0,30 \mathrm{~m}$ et $0,70 \mathrm{~m}$ de la surface, plus un certain nombre de points tous les mètres jusqu'au fond. M. Fouillet précise également que le dispositif de mesure installé sur la Rivière Neuve comporte un transporteur aérien en vue de son fonctionnement en période de crue. Des mesures de nuit pourront ètre nécessaires pour ne pas gêner la navigation ou être gènées par elle, et, éventuellement, pour jauger au maximum de la crue.

M. Hupner rappelle qu'en dehors des essais qu'elle fit pendant la guerre au barrage de Poses, la Compagnie Centrale d'Energie Electrique avait fait, il y a quelque trente ans, au temps où elle avait demandé la concession de la chute de ce barrage, des études systématiques de débit, de remous sur le bief amont et de propagation des intumescences sur le bief aval à la suite des lâchures brusques qui pouvaient être faites par l'usine.

Répondant à une question de M. Rrmenienas, M. FouiLLET indique que les tarages de la base de ChatouBougival seront faits au moyen de deux échelles pour les débits supérieurs à $150 \mathrm{~m}^{3} / \mathrm{s}$ qui donnent une pente de lordre de quelques centimètres par kilomètre, suffisante pour appliquer le principe des stations à deux échelles (1); pour les débits inférieurs à $150 \mathrm{~m} 3 / \mathrm{s}$, ce sera au contraire la hauteur de la lame déversante au barrage de Chatou qui permettra de caleuler le débit, compte tenu de la part qui passe par le bras de Bougival.

M. Remenienas rappelle, a ce sujet, qu'a Poses, sur la Seine, étant donné la difficulté d'installer une station à deux échelles sur ce bief de très faible pente, on avait tenté de relier le débit à la cote du plan d'eau et à une vitesse locale mesurée par moulinet.

M. Founlest précise que les premiers résultats des études faites à Poses de 1942 à 1944 ont été d'obtenir une relation entre le débit et la pente générale du bief, la hauteur étant supposée constante et prise égale à la cote amont du barrage. Malheureusement les expériences n'ont pu être faites que dans une plage assez limitée, c'est pourquoi il $y$ a tout intérêt $\dot{a}$ les reprendre.

M. Remenieras estime que les résultats de Poses ont été difficilement exploitables parce qu'on n'a mesuré qu'une seule vitesse locale, et qu'ils auraient été meilleurs si on avait relié le débit à une cote d'eau et a une vitesse moyenne calculée à partir des indications de plusieurs moulinets répartis dans la section. Il convient toutefois de noter que les stations à deux échelles ne permettent pas une mesure précise du débit en régime non permanent (2).

M. Labaye remarque que la connaissance de $\partial z / \partial t$ d'après les limnigraphes, donne une idée de la nonpermanence et permel même de calculer une correction a apporter au débit. 'Toutefois, cette correction doit ètre très faible dans le cas d'un cours d'eau comme la Seine.

M. Remenieras pense que sur la seine la non-permanence de l'écoulement n'intervient que par la variation des conditions aux limites aval qu'elle peut provoquer. Autrement dit, le remous causé au droit de l'échelle par l'onde de crue qui se propage vers l'aval donne des conditions aux limites aval variables. Il faut que les pentes de régime permanent soient très faibles pour que leffet d'accélération proprement dit (termes en $\partial \mathrm{Q} / \partial t$. $\partial h / \partial t$, etc.) soit notable.

M. le président félicite M. Fouluser et remercie II. BLosser de cette intéressante communieation qui donne des espérances pour l'avenir.

(1) Voir G. Resenreras : L'Hydraulique des stations limnimétriques pour la mesure des débits des colts d'eau. (Annuaire Hydrologique de la France, 1949. 